\title{
Baseline patient reported outcomes data shows high prevalence of overactive bladder, sexual dysfunction, depression and anxiety in Canadian men with newly diagnosed localized prostate cancer
}

\author{
Henry Han-I Yao ${ }^{1,2,3}$, Robert Trafford Crump ${ }^{1}$, Camille Charbonneau ${ }^{1}$, Asher Khan ${ }^{1}$, Carly Barton ${ }^{1}$, \\ Hilary Brotherhood $^{1,2}$, Jing Jiang ${ }^{1}$, Kevin V. Carlson ${ }^{1,2}$, Richard J. Baverstock ${ }^{1,2}$ \\ ${ }^{1}$ Vesia (Alberta Bladder Centre), Calgary, Alberta, Canada; ${ }^{2}$ Department of Surgery, University of Calgary, Calgary, Alberta, Canada; ${ }^{3}$ Eastern Health \\ Clinical School, Monash University, Melbourne, Australia \\ Contributions: (I) Conception and design: R Baverstock, RT Crump; (II) Administrative support: C Barton, J Jiang, RT Crump; (III) Provision of \\ study materials or patients: R Baverstock, K Carlson; (IV) Collection and assembly of data: C Charbonneau, A Khan, C Barton; (V) Data analysis and \\ interpretation: HHI Yao, RT Crump, R Baverstock, K Carlson, J Jiang, H Brotherhood; (VI) Manuscript writing: All authors; (VII) Final approval of \\ manuscript: All authors. \\ Correspondence to: Dr. Henry Han-I Yao. Vesia (Alberta Bladder Centre), 6601, 7007 14th Street SW, Calgary, AB T2V 1P9, Canada. \\ Email: henry.yao@monash.edu.
}

Background: Research on prostate cancer survivorship patients has largely been on oncological outcome, incontinence and erectile dysfunction, with less data on the relationship between prostate cancer, bladder function and mental health. This study aims to elucidate the prevalence of lower urinary tract symptoms (LUTS), overactive bladder (OAB), sexual dysfunction, depression and anxiety in Canadian men with newly diagnosed localised prostate cancer.

Methods: This is a single-centre prospective cross-sectional study of men with newly diagnosed localized prostate cancer recruited from June 2017 to July 2018. The patient-reported outcomes (PRO) instruments used in this study included the international prostate symptoms score (IPSS), OAB-V8, EQ-5D ${ }^{\mathrm{TM}}$, and the Expanded Prostate Cancer Index Composite short form (EPIC-26). Clinico-pathological data were extracted from medical records. The prevalence of LUTS, OAB, sexual dysfunction, depression and anxiety were determined from the PROs.

Results: A total of 83 patients were included in this study. The median age was 63 . Based on IPSS scores, $55.3 \%$ of men had mild LUTS, 36.8\% had moderate LUTS and 7.9\% had severe LUTS. Based on OAB-V8 scores, $55.8 \%$ of men had a score of 8 or higher, suggestive of OAB. Only 55.8\% of men reported erections adequate for intercourse. $23.1 \%$ of men reported to have a moderate to big problem with depression, and $28.8 \%$ of men reported to have a degree of anxiety or depression.

Conclusions: OAB is a significant problem in men with newly diagnosed localized prostate cancer, with a prevalence of $55.8 \%$ based on this study. Baseline sexual dysfunction, anxiety and depression are also prevalent in this population.

Keywords: Erectile dysfunction; epidemiology; lower urinary tract symptoms (LUTS); prostate neoplasms; urinary bladder; overactive

Submitted Mar 06, 2020. Accepted for publication Aug 23, 2020.

doi: 10.21037/tau-20-689

View this article at: http://dx.doi.org/10.21037/tau-20-689 


\section{Introduction}

Prostate cancer is the second most commonly diagnosed cancer in men around the globe and the most commonly diagnosed non-skin cancer in Canadian men $(1,2)$. With 1 in 7 men diagnosed with prostate cancer in their lifetime, there is a large cohort of cancer survivorship patients in the society (1). Research in the prostate cancer survivorship cohort have largely been centred around oncological outcome followed by urinary incontinence and erectile dysfunction (3). There are less studies available examining the relationship between prostate cancer and its treatment on bladder function (3).

Bladder dysfunction can be subdivided into overactive bladder $(\mathrm{OAB})$ and underactive bladder (UAB) syndrome. $\mathrm{OAB}$ syndrome is defined by the international continence society (ICS) as "urinary urgency, usually accompanied by frequency and nocturia, with or without urgency urinary incontinence, in the absence of urinary tract infection (UTI) or other obvious pathology" (4). The prevalence of OAB in population-based survey of men from multiple studies ranged from $10.8 \%$ to $27.2 \%(5-9)$. Canadian based studies revealed a prevalence of $\mathrm{OAB}$ in $13.1 \%$ to $14.8 \%$ of adult men $(5,7)$.

There are many studies in the literature examining the risk of prostate cancer in patients with lower urinary tract symptoms (LUTS) $(10,11)$. However, there are few studies specifically examining the prevalence of LUTS prior to prostate cancer treatment. One of those study examining men diagnosed with localised prostate cancer, reported a LUTS rate of $50.1 \%$ in Japanese men and $46.9 \%$ in American men, using AUA symptom score of $\geq 8$ as a cutoff for LUTS (12). This is in comparison to the prevalence of moderate to severe LUTS in the general Japanese and American population of $36.6 \%$ and $25.5 \%$, respectively (13). Namiki et al. reported a mean AUA symptom score to be 9.1 and 8.7 in Japanese and American men with localised prostate cancer, respectively (12). Rees et al. reported a mean IPSS score of 11.6 in men with locally advanced or metastatic prostate cancer, which is compared with 4.4 in control men in the study (14). Two other large studies examined the rates of storage LUTS in men with newly diagnosed localised prostate cancer. The Protec $T$ study group found baseline rates of storage LUTS to be common with $33 \%$ of men suffering from frequency and $22 \%$ from nocturia (15). Similarly, the CEASAR study reported the rates of urinary frequency to be $19-24 \%$ (16). None of these studies specifically examined the rates of $\mathrm{OAB}$ in prostate cancer patients prior to any treatment.

There are only a few studies examining the rates of depression and anxiety in prostate cancer patients despite both being common amongst cancer patients and can have adverse correlation with treatment outcomes (17). A previous meta-analysis has demonstrated a high rate of depression and anxiety pre-treatment, on-treatment and post-treatment (17). The ProtecT study group published their findings after the meta-analysis and reported depression rates of $6 \%$ and anxiety rates of $20 \%$ in men with newly diagnosed localised prostate cancer (15). Previous published Canadian studies have only examined the prevalence of depression and anxiety post-treatment, and one study reported suicidal ideation to be as high as $14 \%(17,18)$.

It is important to manage quality of life issues for men with prostate cancer during their prolonged cancer survivorship. This begins with a better understanding of the baseline demographics. Therefore, this study aims to elucidate the prevalence of LUTS, OAB, sexual dysfunction, depression and anxiety in Canadian men with newly diagnosed localised prostate cancer before initiation of treatment. We present the following article in accordance with the STROBE reporting checklist (available at http:// dx.doi.org/10.21037/tau-20-689).

\section{Methods}

This is a prospective cross-sectional study of Canadian men with newly diagnosed localized prostate cancer. Participants were recruited from the Prostate Cancer Centre in Calgary, Canada-a metropolitan area of approximately 1.4 million residents. The Prostate Cancer Centre offers an information seminar for men newly diagnosed with localized prostate cancer. At the seminar, patients were offered the option to participate in this research study. Those who met the eligibility criteria were provided with further details and a written informed consent was obtained. The protocol for this study was approved by the Health Research Ethics Board of Alberta (HREBA.CC-16-0850). This study conformed to the provisions of the Declaration of Helsinki (as revised in 2013) (19). All patients enrolled completed the informed consent form.

Patients were recruited from the period of June 2017 to July 2018. Men were eligible for this study if they were 18 years of age or older, spoke English or had a translator available, had not been previously diagnosed with prostate cancer, and had not yet started prostate cancer treatment. 
Table 1 Demographics of this study cohort of newly diagnosed prostate cancer patients

\begin{tabular}{lcc}
\hline Variables & Values & $\mathrm{N}$ \\
\hline Total number of patients & 83 & \\
Median age (years) & $63($ IQR $59-67)$ & 83 \\
Median PSA (ng/mL) & 6.7 (IQR 4.8-9.6) & 82 \\
Median prostate volume $(\mathrm{mL})$ & 35.3 (IQR 26.7-43.1) & 82 \\
Median PSA density & $0.20($ IQR $0.15-0.29)$ & 81 \\
Digital rectal examination findings & & \\
Abnormal & $14.5 \%$ & 12 \\
Normal & $62.7 \%$ & 52 \\
Unknown & $22.9 \%$ & 19 \\
ISUP Grade Group & & \\
1 & $36.1 \%$ & 30 \\
2 & $37.3 \%$ & 31 \\
3 & $12.0 \%$ & 10 \\
4 & $10.8 \%$ & 9 \\
5 & $3.6 \%$ & 3 \\
\hline
\end{tabular}

PSA, prostate specific antigen; ISUP, International Society of Urological Pathology; N, number of patients; IQR, interquartile range.

Individuals were excluded if they had previously been diagnosed with prostate cancer, if metastatic disease was suspected, or if they were unwilling to adhere to the data collection protocol. A PSA cut-off level was not used as an exclusion criterion provided the patient had no metastatic disease (T1-4N0-1M0) on staging scans. Potential participants were offered an incentive of $\$ 20-\$ 30$ if they completed the entire study.

Patients included in this study were sent a link to a secure website to complete their intake survey (a postal mail option was available for patients without internet access). This intake survey included: (I) demographic data, (II) medical history information, and (III) patient-reported outcome (PRO) instruments. The PRO instruments included the international prostate symptoms score (IPSS) (20), OAB-V8 (21), EQ-5DTM (22), and the Expanded Prostate Cancer Index Composite short form (EPIC-26) (23). An $\mathrm{OAB}-\mathrm{V} 8$ global score of 8 or higher indicates the possibility of OAB (21). Patients with OAB-V8 global score of 8 or higher were offered a urodynamics study to further evaluate their $\mathrm{OAB}$.
Clinicopathological data were extracted from participants' medical records by a trained data enterer. All data were collected and managed using REDCap electronic database securely hosted by The University of Calgary's Clinical Research Unit $(24,25)$.

\section{Statistical analysis}

Descriptive analyses were performed to describe the epidemiological findings from each PRO instrument used. Missing data due to incomplete questionnaires on followup are reported. Univariate analyses were performed to determine if age, PSA, prostate volume, body mass index (BMI), IPSS, presence and absence of intraductal prostate cancer, ISUP grade group, and location of tumour were predictor of $\mathrm{OAB}$ based on $\mathrm{OAB}-\mathrm{V} 8$ scores. Median values were compared using Mann-Whitney $U$ test. Proportions were compared using Chi-squared test or Fisher's exact test. Multivariate analyses were performed using binomial logistic regression. Statistical significance was defined as $\mathrm{P}$ value of $<0.05$. Statistical analysis was performed using SPSS Version 25.0 (Armonk, NY: IBM Corp.).

\section{Results}

\section{Demographics}

A total of 83 patients with newly diagnosed prostate cancer were included in this study. The baseline demographics of our cohort are outlined in Table 1.

\section{IPSS and $O A B V 8$ findings}

The median IPSS score was 6 (IQR 3-12, n=76). Of the 76 patients who returned their IPSS questionnaire, 55.3\% $(\mathrm{n}=42)$ of patients had mild LUTS, 36.8\% ( $\mathrm{n}=28)$ moderate LUTS, 7.9\% ( $\mathrm{n}=6)$ severe LUTS. The median OAB-V8 score was 8 (IQR $5.3-14, n=52$ ). Of the 52 men who returned their $\mathrm{OAB}-\mathrm{V} 8$ questionnaire, the proportion of men with $\mathrm{OAB}-\mathrm{V} 8$ score greater or equal to 8 was $55.8 \%$ $(n=29)$, and OAB-V8 score less than 8 was $44.2 \%(n=23)$.

\section{Predictors of higher $\mathrm{OAB}-\mathrm{V} 8$ scores}

Univariate analyses revealed IPSS and PSA to be the only predictor of OAB on OAB-V8 (Table 2). This is consistent with the finding that IPSS correlates well with OAB-V8 with a Spearman's rho correlation coefficient of 0.753 
Table 2 Univariate analysis of predictors of overactive bladder based on OAB-V8 scores

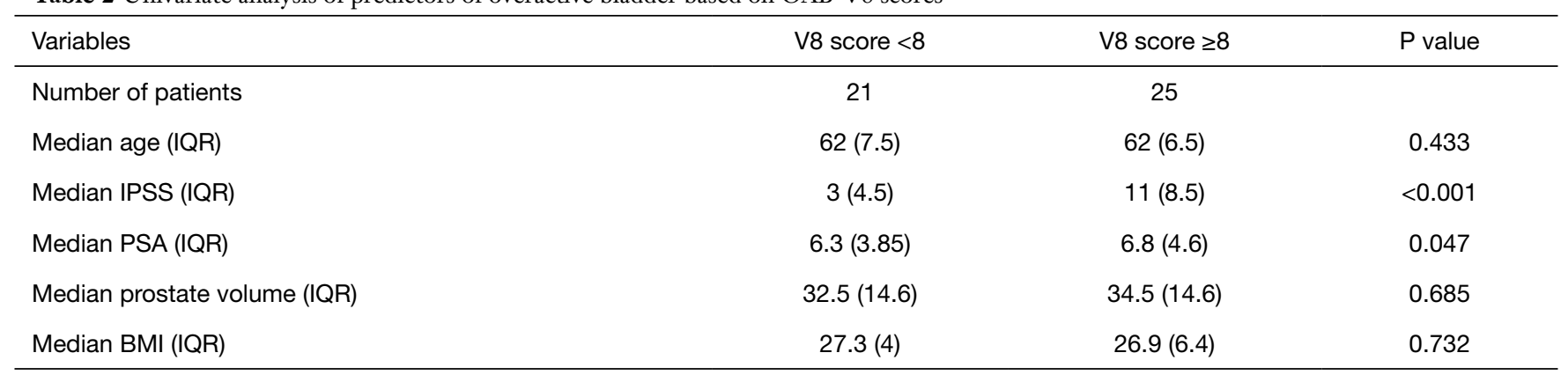

IQR, interquartile range; IPSS, international prostate symptom score; PSA, prostate specific antigen; BMI, body mass index.

$(\mathrm{P}<0.001)$. The rate of $\mathrm{OAB}$ was not statistically different for patients with intraductal prostate cancer compared with those without ( $70 \%$ vs. $50 \%, \mathrm{P}=0.31$, Fisher's exact test). Similarly, the rates of OAB did not differ in patients with high (ISUP 4 and 5), intermediate (ISUP 2 and 3) and low risk (ISUP 1) prostate cancer $(33.3 \%, 61.5 \%, 55 \%, \mathrm{P}=0.53)$. The rates of $\mathrm{OAB}$ was also not different for presence of prostate cancer in different parts of the prostate gland (apex, mid-gland and base). On multivariate analysis, only IPSS was a statistically significant predictor of $\mathrm{OAB}(\mathrm{P}=0.002)$.

\section{EPIC-26}

The EPIC-26 questionnaire was completed by 52 patients. In regard to the urinary function domains, $73.1 \%(\mathrm{n}=38)$ of men rarely or never leaked urine and $26.9 \%(n=14)$ experienced leakage of urine ranging from once a day to once a week. Similarly, $75 \%(n=39)$ of men reported total control of urination, $25 \%(n=13)$ experienced a degree of dribbling of urine. None of the men used any adult diapers. The majority of patients reported no problem with pain or burning on urination $(90.4 \%, \mathrm{n}=47)$, nor bleeding with urination $(96.2 \%, \mathrm{n}=50)$. A more significant number of men reported storage and voiding LUTS, including $63.5 \%$ $(n=33)$ of men whom reported to have weak urine stream or incomplete emptying, $40.4 \%(n=21)$ of men whom reported to have dripping or leaking urine, and $63.5 \%(n=33)$ of men whom reported to have frequency of urination. Overall, $40.4 \%(n=21)$ of men rated no problem with their urinary function, $42.3 \%(n=22)$ rated it to be a very small problem, $11.5 \%(\mathrm{n}=6)$ rated it to be a small problem, and only $5.8 \%$ $(\mathrm{n}=3)$ rated it to be a moderate problem.

In regard to the sexual function domain, $11.5 \%(n=6)$ of men reported to have very poor to no erections at time of diagnosis, $15.4 \%(n=8)$ of men had poor erections, $30.8 \%$ of men had fair erections, $17.3 \%$ ( $n=9)$ of men had good erections, and $25 \%(n=13)$ of men had very good erections. The ability to reach orgasm was very poor to none in $9.6 \%$ $(n=5)$ of men, poor in $9.6 \%(n=5)$ of men, fair in $21.2 \%$ $(n=11)$ of men, good in $26.9 \%(n=14)$ of men, and very good in $32.7 \%(n=17)$ of men. The quality of erections was firm enough for intercourse in $55.8 \%(\mathrm{n}=29)$ of men, firm enough for masturbation and foreplay only in $26.9 \%(n=14)$ of men, not firm enough for any sexual activity in $11.5 \%$ $(\mathrm{n}=6)$ of men and absent altogether in $5.8 \%(\mathrm{n}=3)$ of men. Overall, men rated their ability to function sexually as very good in $21.2 \%(\mathrm{n}=11)$, good in $26.9 \%(\mathrm{n}=14)$, fair in $25 \%$ $(n=13)$, poor in $17.3 \%(n=9)$, and very poor in $9.6 \%(n=5)$ of men. Overall men rated their sexual function to be a big problem in $5.8 \%(\mathrm{n}=3)$, a moderate problem in $15.4 \%(\mathrm{n}=8)$, a small problem in $11.5 \%(\mathrm{n}=6)$, a very small problem in $26.9 \%(n=14)$, and no problem in $40.4 \%(n=21)$ of men.

In regard to mental function domain, $7.7 \%(n=4)$ of men reported to have a big problem with feeling depressed, $15.4 \%(n=8)$ of men reported to have a moderate problem, $11.5 \%(\mathrm{n}=6)$ of men reported to have a small problem, and $65.4 \%(n=34)$ reported to have a very small problem with depression.

\section{Generic bealth and mental status-EQ-5DTM}

Of the 52 patients who filled in their EQ-5DTM questionnaire, the median overall health status was 80 (IQR 72.8-90). The majority of patients reported no problems with mobility $(86.5 \%, \mathrm{n}=45)$, no problems with selfcare $(98.1 \%, \mathrm{n}=51)$ and no problems with usual activities $(90.4 \%, n=47)$. Most patients did not report any pain or discomfort $(69.2 \%, n=36), 23.1 \%(n=12)$ reported slight pain or discomfort, $5.8 \%(\mathrm{n}=3)$ reported moderate pain or discomfort, and $1.9 \%(n=1)$ reported extreme pain 
or discomfort. Most patients did not report anxiety or depression $(71.2 \%, n=37)$. However, $17.3 \%(n=9)$ of men did report that they were slightly anxious or depressed, and $11.5 \%(n=6)$ reported that they were moderately anxious or depressed.

\section{Discussion}

This study found LUTS and OAB to be a significant problem affecting half of Canadian men with newly diagnosed localised prostate cancer. We found that $44.7 \%$ of men had moderate or severe LUTS based on IPSS, and $55.8 \%$ of men had likely OAB based on OAB-V8 score. The rate of $\mathrm{OAB}$ in our study cohort is significantly higher than the $14.8 \%$ rate of $\mathrm{OAB}$ found in a Canadian population study looking at people aged 35 and over (5). This remains true even if only comparing with the older subgroup of men in the study by Corcos et al. (5), as the subgroup analysis found the prevalence of urge urinary incontinence and $\mathrm{OAB}$ to be $7.5 \%, 21.5 \%$ and $29.5 \%$ for men aged $55-64$, $65-74$, and 75 and above, respectively. A similar rate of $\mathrm{OAB}$ has been reported by another Canadian population study which found the prevalence of $\mathrm{OAB}$ symptoms to be $13.1 \%$ in men aged 18 and above (7). The subgroup analysis of the previous study found men aged 41-64 and $\geq 65$ to have a prevalence of $\mathrm{OAB}$ symptoms of $17.5 \%$ and $14.9 \%$, respectively (7).

The rates of LUTS and OAB is likely higher in our study population because men with urinary symptoms are more likely to seek healthcare advice and be diagnosed with their underlying prostate cancer (26). This is supported by a previous study which has shown that men treated for LUTS were more likely to undergo prostate biopsy (11). Furthermore, a study of Japanese and American men has found a similarly higher rate of moderate or severe LUTS on IPSS of 50.1\% in Japanese men and 46.9\% in American men (12). Studies that have performed urodynamic studies prior to radical prostatectomy have demonstrated detrusor overactivity (DO) rates to be $18.7-25.5 \%(27,28)$. PRO scores prior to UDS were not available in these studies. Given that DO is found in $76.1 \%$ of men with $\mathrm{OAB}$ symptoms (29), it is likely that the prevalence of $\mathrm{OAB}$ symptoms is higher than the reported DO rates in studies of UDS pre-radical prostatectomy. It is important to recognise the significant rates of LUTS and OAB in prostate cancer patients, as it may need to be treated prior to radiotherapy and addressed following radical prostatectomy to ensure it is not contributing to a poorer continence outcome.
We found IPSS to be the only predictor of OAB defined using OAB-V8. The two score also strongly correlated with each other. This is not unexpected as IPSS has many components that are also tested in OAB-V8 such as frequency, urgency and nocturia. We have previously published our cross-walk modelling of OAB-V8 score from IPSS score (30). Apart from IPSS, there were no other factors that were predictive of OAB on OAB-V8. Previous studies have shown $\mathrm{OAB}$ to be more prevalent with increasing age (5). However, given the similar age of our patient cohort with an interquartile range of 59-67, age becomes a less important factor in predicting OAB. ISUP grade group and location of tumour in apex, mid-gland or base does not influence rates of OAB. This is expected as prostate cancer tends to be located in the peripheral zone and therefore unlikely to contribute to urinary symptoms, especially for localised prostate cancer.

This study shows that it is not uncommon for men diagnosed with localised prostate cancer to already have poor erections prior to any treatment. We found that $26.9 \%$ of men had poor or no erections, and $19.2 \%$ of men had poor or no ability to reach orgasm. Only $55.8 \%$ of men reported erections adequate for intercourse and $26.9 \%$ rated their overall ability to function sexually as poor or very poor. This is similar to a study by Briganti et al. which showed that $46 \%$ of patients had no ED pre-operatively, $19.5 \%$ had mild ED, $8.3 \%$ had mild to moderate ED, $7.4 \%$ had moderate ED and $18.9 \%$ had severe ED based on International Index of Erectile Function (IIEF) scores (31). Another study showed that $21.2 \%$ of patients reported an moderate or severe ED based in IIEF scores pre-operatively (32). These results are also very similar to those published by Salonia et al. which showed that $43 \%$ of patients had normal erections pre-op, $13 \%$ had mild ED, $8 \%$ had mild to moderate ED, $8 \%$ had moderate ED and $28 \%$ had severe ED based on IIEF (33).

The prevalence of anxiety and depression amongst men diagnosed with prostate cancer is not insignificant. We found that $28.8 \%$ of men in this study reported to be slightly or moderately anxious or depressed based on EQ5D questionnaire. Similarly, $22.9 \%$ of men reported to have a moderate or big problem with feeling depressed based on the EPIC questionnaire. This is not dissimilar to the meta-analysis which revealed pre-treatment depression prevalence rate of $17.27 \%$ and pre-treatment anxiety prevalence rates to be $27.04 \%$ (17). It is important to screen for and recognise the presence of anxiety and depression pre-operatively, and to provide appropriate psychological 
support and treatment to improve the overall holistic patient care.

This study has several limitations including the relatively small sample size of this study. Despite the small sample size, to our knowledge this is the first study reporting baseline OAB-V8 scores in men with localised prostate cancer. Another limitation is that not all patient responded to questionnaires, with $91.6 \%$ responded to IPSS, and $62.7 \%$ responded to OAB-V8, EPIC-26 and EQ-5DTM. The authors acknowledge that there is a possibility that men with more symptoms are more likely to participate in the study and completion of questionnaires. However, there is no incentive for men to do so as treatment regimen is not a part of the study. The questionnaires were offered to all patient who agreed initially to participate in this study, hence the dropout of questionnaires is likely to be random. The use of medications for treatment of LUTS and $\mathrm{OAB}$ were no uniformly reported and therefore not included in the result of the study. This may have resulted in an underestimation of the true prevalence of $\mathrm{OAB}$ as mild cases may have resolved with use of these medications. Finally, as this is a single centre study in Canada, clinicians should understand the limits of generalizing our findings, especially for men outside of Canada.

\section{Conclusions}

$\mathrm{OAB}$ is a significant problem in men with newly diagnosed localized prostate cancer, with a prevalence of $55.8 \%$ based on this study. Baseline sexual dysfunction in this population of men is also significant with only half of the men able to achieve an erection adequate for intercourse. Anxiety and depression also affect a quarter of these men. It is important to identify these pre-operatively as it can impact on management decisions and counselling of patients on functional outcome. With increasing focus on cancer survivorship, adequate treatment of these issues starting pre-treatment can potentially improve the quality of life for men post-prostate cancer therapy.

\section{Acknowledgments}

The authors would like to thank Dave Bateman and the Prostate Cancer Centre in Calgary, AB for their assistance with identifying potential study participants.

Funding: This work was awarded by Prostate Cancer Canada, Grant \#D2016-1353, and is proudly funded by the Movember Foundation.

\section{Footnote}

Reporting Checklist: The authors have completed the STROBE reporting checklist. Available at http://dx.doi. org/10.21037/tau-20-689

Data Sharing Statement: Available at http://dx.doi. org/10.21037/tau-20-689

Conflicts of Interest: All authors have completed the ICMJE uniform disclosure form (available at http://dx.doi. org/10.21037/tau-20-689). The authors have no conflicts of interest to declare.

Etbical Statement: The authors are accountable for all aspects of the work in ensuring that questions related to the accuracy or integrity of any part of the work are appropriately investigated and resolved. The protocol for this study was approved by the Health Research Ethics Board of Alberta (HREBA.CC-16-0850). This study conformed to the provisions of the Declaration of Helsinki (as revised in 2013) (19). All patients enrolled completed the informed consent form.

Open Access Statement: This is an Open Access article distributed in accordance with the Creative Commons Attribution-NonCommercial-NoDerivs 4.0 International License (CC BY-NC-ND 4.0), which permits the noncommercial replication and distribution of the article with the strict proviso that no changes or edits are made and the original work is properly cited (including links to both the formal publication through the relevant DOI and the license). See: https://creativecommons.org/licenses/by-nc-nd/4.0/.

\section{References}

1. Fradet Y, Klotz L, Trachtenberg J, et al. The burden of prostate cancer in Canada. Can Urol Assoc J 2009;3:S92-S100.

2. Ferlay J, Soerjomataram I, Dikshit R, et al. Cancer incidence and mortality worldwide: sources, methods and major patterns in GLOBOCAN 2012. Int J Cancer 2015;136:E359-86.

3. Thiruchelvam N, Cruz F, Kirby M, et al. A review of detrusor overactivity and the overactive bladder after radical prostate cancer treatment. BJU Int 2015;116:853-61.

4. Bo K, Frawley HC, Haylen BT, et al. An International Urogynecological Association (IUGA)/International 
Continence Society (ICS) joint report on the terminology for the conservative and nonpharmacological management of female pelvic floor dysfunction. Neurourol Urodyn 2017;36:221-44.

5. Corcos J, Schick E. Prevalence of overactive bladder and incontinence in Canada. Can J Urol 2004;11:2278-84.

6. Coyne KS, Sexton CC, Vats V, et al. National community prevalence of overactive bladder in the United States stratified by sex and age. Urology 2011;77:1081-7.

7. Herschorn S, Gajewski J, Schulz J, et al. A populationbased study of urinary symptoms and incontinence: the Canadian Urinary Bladder Survey. BJU Int 2008;101:52-8.

8. Irwin DE, Milsom I, Hunskaar S, et al. Population-based survey of urinary incontinence, overactive bladder, and other lower urinary tract symptoms in five countries: results of the EPIC study. Eur Urol 2006;50:1306-14; discussion 14-5.

9. Stewart WF, Van Rooyen JB, Cundiff GW, et al. Prevalence and burden of overactive bladder in the United States. World J Urol 2003;20:327-36.

10. Oh JJ, Jeong SJ, Jeong CW, et al. Is there any association between the severity of lower urinary tract symptoms and the risk of biopsy-detectable prostate cancer in patients with PSA level below $20 \mathrm{ng} / \mathrm{ml}$ in multi-core prostate biopsy? Prostate 2013;73:42-7.

11. Weight CJ, Kim SP, Jacobson DJ, et al. The effect of benign lower urinary tract symptoms on subsequent prostate cancer testing and diagnosis. Eur Urol 2013;63:1021-7.

12. Namiki S, Litwin MS, Kwan L, et al. Association of lower urinary tract symptoms with sexual dysfunction: a crosscultural study between Japanese and American men with localized prostate cancer. BJU Int 2009;104:1071-6.

13. Tsukamoto T, Kumamoto Y, Masumori N, et al. Prevalence of prostatism in Japanese men in a communitybased study with comparison to a similar American study. J Urol 1995;154:391-5.

14. Rees J, Waldron D, O'Boyle C, et al. Prospective vs retrospective assessment of lower urinary tract symptoms in patients with advanced prostate cancer: the effect of 'response shift'. BJU Int 2003;92:703-6.

15. Lane A, Metcalfe C, Young GJ, et al. Patient-reported outcomes in the Protec T randomized trial of clinically localized prostate cancer treatments: study design, and baseline urinary, bowel and sexual function and quality of life. BJU Int 2016;118:869-79.

16. Resnick MJ, Barocas DA, Morgans AK, et al. Contemporary prevalence of pretreatment urinary, sexual, hormonal, and bowel dysfunction: Defining the population at risk for harms of prostate cancer treatment. Cancer 2014;120:1263-71.

17. Watts S, Leydon G, Birch B, et al. Depression and anxiety in prostate cancer: a systematic review and meta-analysis of prevalence rates. BMJ Open 2014;4:e003901.

18. Rice SM, Oliffe JL, Kelly MT, et al. Depression and Prostate Cancer: Examining Comorbidity and MaleSpecific Symptoms. Am J Mens Health 2018;12:1864-72.

19. World Medical Association. World Medical Association Declaration of Helsinki: ethical principles for medical research involving human subjects. JAMA 2013;310:2191-4.

20. Barry MJ, Fowler FJ Jr, O'Leary MP, et al. The American Urological Association symptom index for benign prostatic hyperplasia. The Measurement Committee of the American Urological Association. J Urol 1992;148:154957; discussion 64.

21. Coyne KS, Zyczynski T, Margolis MK, et al. Validation of an overactive bladder awareness tool for use in primary care settings. Adv Ther 2005;22:381-94.

22. Desroziers K, Aballea S, Maman K, et al. Estimating EQ-5D and OAB-5D health state utilities for patients with overactive bladder. Health Qual Life Outcomes 2013;11:200.

23. Wei JT, Dunn RL, Litwin MS, et al. Development and validation of the expanded prostate cancer index composite (EPIC) for comprehensive assessment of health-related quality of life in men with prostate cancer. Urology 2000;56:899-905.

24. Harris PA, Taylor R, Thielke R, et al. Research electronic data capture (REDCap)--a metadata-driven methodology and workflow process for providing translational research informatics support. J Biomed Inform 2009;42:377-81.

25. Harris PA, Taylor R, Minor BL, et al. The REDCap consortium: Building an international community of software platform partners. J Biomed Inform 2019;95:103208.

26. Martin RM, Vatten L, Gunnell D, et al. Lower urinary tract symptoms and risk of prostate cancer: the HUNT 2 Cohort, Norway. Int J Cancer 2008;123:1924-8.

27. Barnoiu OS, Vozmediano-Chicharro R, Garcia-Galisteo $\mathrm{E}$, et al. Urodynamic assessment of bladder and urethral sphincter function before and after robot-assisted radical prostatectomy. Actas Urol Esp 2014;38:78-83.

28. Matsukawa Y, Hattori R, Komatsu T, et al. De novo detrusor underactivity after laparoscopic radical prostatectomy. Int J Urol 2010;17:643-8. 
29. Al-Ghazo MA, Ghalayini IF, Al-Azab R, et al. Urodynamic detrusor overactivity in patients with overactive bladder symptoms. Int Neurourol J 2011;15:48-54.

30. Trafford Crump R, Sehgal A, Wright I, et al. From Prostate Health to Overactive Bladder: Developing a Crosswalk for the IPSS to OAB-V8. Urology 2019;125:73-8.

31. Briganti A, Gallina A, Suardi N, et al. Predicting erectile function recovery after bilateral nerve sparing radical

Cite this article as: Yao HHI, Crump RT, Charbonneau C, Khan A, Barton C, Brotherhood H, Jiang J, Carlson KV, Baverstock RJ. Baseline patient reported outcomes data shows high prevalence of overactive bladder, sexual dysfunction, depression and anxiety in Canadian men with newly diagnosed localized prostate cancer. Transl Androl Urol 2020;9(5):2046-2053. doi:10.21037/tau-20-689 prostatectomy: a proposal of a novel preoperative risk stratification. J Sex Med 2010;7:2521-31.

32. Novara G, Ficarra V, D'Elia C, et al. Preoperative criteria to select patients for bilateral nerve-sparing roboticassisted radical prostatectomy. J Sex Med 2010;7:839-45.

33. Salonia A, Zanni G, Gallina A, et al. Baseline potency in candidates for bilateral nerve-sparing radical retropubic prostatectomy. Eur Urol 2006;50:360-5. 